\title{
Pityriasis rubra pilaris in Kombination mit Pseudoakanthosis nigricans
}

\section{Pityriasis rubra pilaris in Combination with Acanthosis nigricans}

Autoren

Institut
G. Haas, P. von den Driesch

Klinik für Dermatologie und Allergologie, Zentrum für Hautkrankheiten, Klinikum Stuttgart
Bibliografie

DOI http://dx.doi.org/

10.1055/s-0032-1310135

Online-Publikation: 7.8.2012

Akt Dermatol 2012; 38: 403-406

(c) Georg Thieme Verlag KG

Stuttgart · New York

ISSN 0340-2541

\section{Korrespondenzaddresse}

Prof. Dr. med. Peter

von den Driesch

Klinik für Dermatologie

und Allergologie

Zentrum für Hautkrankheiten

Klinikum Stuttgart

Priessnitzweg 24

70374 Stuttgart

pdriesch@klinikum-stuttgart.de

\section{Zusammenfassung \\ $\nabla$}

Wir berichten über einen 53-jährigen Patienten, welcher seit mehr als 12 Jahren mit der Diagnose „Psoriasis“ behandelt wurde und sich mit einer Suberythrodermie mit randbetonten Plaques mit diskreter Schuppung betont an Stamm und Extremitäten mit dazwischen liegenden Arealen gesunder Haut präsentierte. Daneben bestand eine Vergröberung des Hautreliefs mit fibrinösen

\section{Einleitung \\ $\nabla$}

Die Pityriasis rubra pilaris ist eine seltene, meist chronisch verlaufende Erkrankung unklarer Ätiologie [6,9], welche durch palmoplantare Hyperkeratosen und follikuläre Keratosen gekennzeichnet ist und sich häufig in Form einer (Sub-)Erythrodermie präsentiert $[1,13]$.

Betroffen sind die Streckseiten der Extremitäten, das Capillitium, das Gesicht und der Rumpf sowie auch Handinnenflächen und Fußsohlen. Die wichtigsten Differenzialdiagnosen sind die klinisch oft nur sehr schwer abgrenzbaren kutanen Lymphome, Ekzeme und Psoriasis [2].

Bei der Acanthosis nigricans handelt es sich um eine Hyperpigmentierung und Papillomatose in den großen Körperfalten. Man unterscheidet dabei grundsätzlich zwischen einer paraneoplastischen Form („Acanthosis nigricans maligna“), besonders häufig assoziiert mit Adenokarzinomen des Magens [8] und verschiedenen nicht-paraneoplastischen Formen („Pseudoacanthosis nigricans“).

\section{Kasuistik \\ $\nabla$}

Ein 53-jähriger Patient $(170 \mathrm{~cm}, 85 \mathrm{~kg})$ stellte sich mit einer „seit mehr als 12 Jahren bekannten Psoriasis“ vor, welche bisher äußerlich mit VitaminD3-Analoga sowie kurzzeitig mit UV-Therapie be-
Papeln und schwarzer Hyperpigmentierung in der Nacken- und Halspartie sowie axillär und inguinal. Klinisch und histologisch diagnostizierten wir sowohl eine Pityriasis rubra pilaris als auch eine Acanthosis nigricans. Wir leiteten eine Systemtherapie mit Methotrexat, eine Lichttherapie mit Bade-PUVA sowie eine intensive Lokaltherapie ein. Eine Kombination dieser beider Erkrankungen wurde in der Literatur bisher nicht beschrieben.

handelt worden sei. Seit etwa einem Jahr sei es zu einer deutlichen Verschlechterung gekommen. Die Infektanamnese war leer, Gelenkbeschwerden verneinte er. Als Vorerkrankungen gab er einen Apoplex im Jahre 2005 an. An Medikamenten nahm er Bisoprolol, ASS, Valsartan sowie ein Kombinationspräparat aus Simvastatin und Ezetimib ein.

Es zeigte sich eine Suberythrodermie mit landkartenartigen randbetonten Plaques mit leichter Schuppung betont an Stamm und Extremitäten mit dazwischen liegenden Arealen gesunder Haut ( Abb. 1 und Abb.2). Daneben auch mäßig ausgeprägte Kopfschuppung. Darüber hinaus war im Nacken, an der Halspartie, axillär sowie inguinal mit Perianalbereich eine Vergröberung des Hautreliefs mit fibrinösen Papeln und schwarzer Hyperpigmentierung zu sehen ( $\bullet$ Abb.3).

Das Labor ergab neben leichtgradig erhöhten Leberwerten ein geringfügig erniedrigtes Kalium, welches daraufhin substituiert wurde. Daneben zeigte sich ein diskret erhöhter HbA1c von 6,1\%. Das übrige Labor, einschließlich Blutfette, Serumelektrophorese sowie sämtlicher üblicher Tumormarker war komplett normwertig.

Die bildgebenden Untersuchungen, Röntgen-Thorax, Abdomensonografie sowie Koloskopie blieben unauffällig/ohne Hinweise auf Malignität.

Wir entnahmen zwei Gewebeproben. Probe 1 $(\bullet$ Abb.4), welche am oberen Rücken entnommen wurde, zeigte histopathologisch eine folliku- 


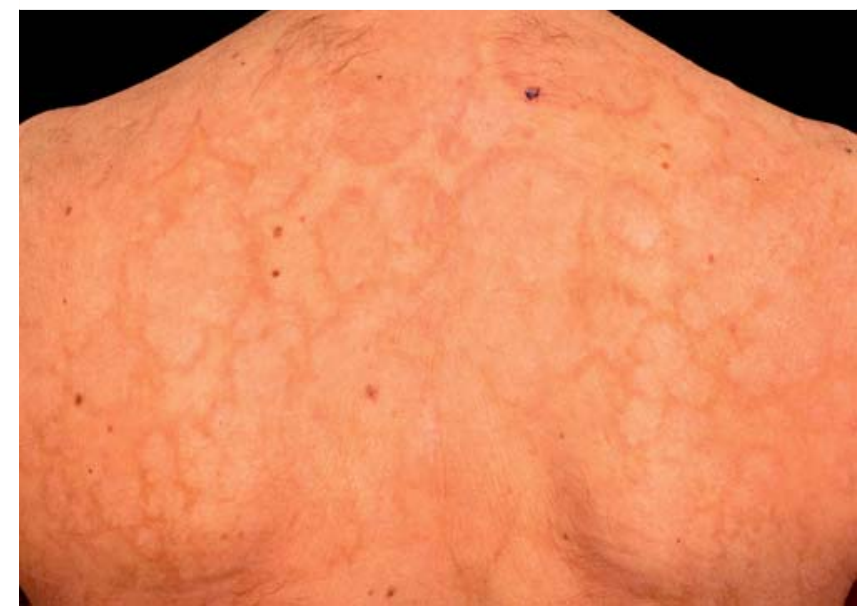

Abb.1 Suberythrodermie mit anulären Herden und Nappes claires bei Pityriasis rubra pilaris.

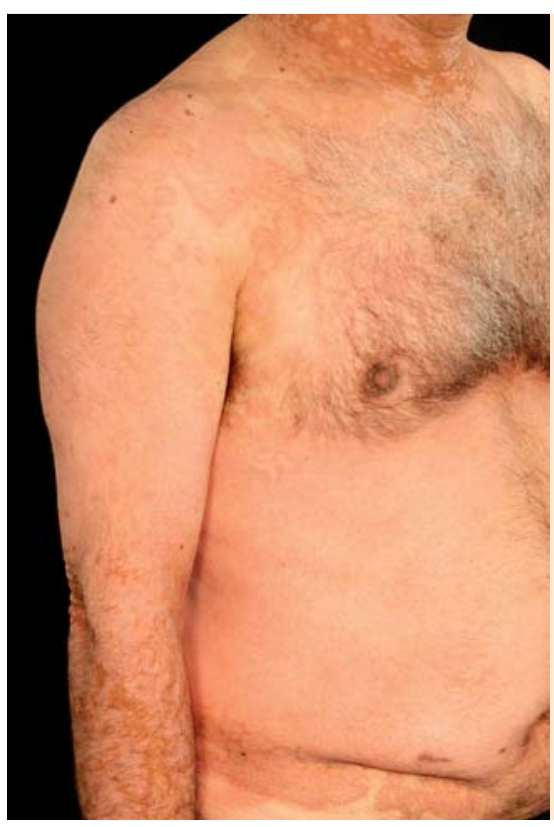

Abb.2 Pityriasis rubra pilaris und Akanthosis nigricans.

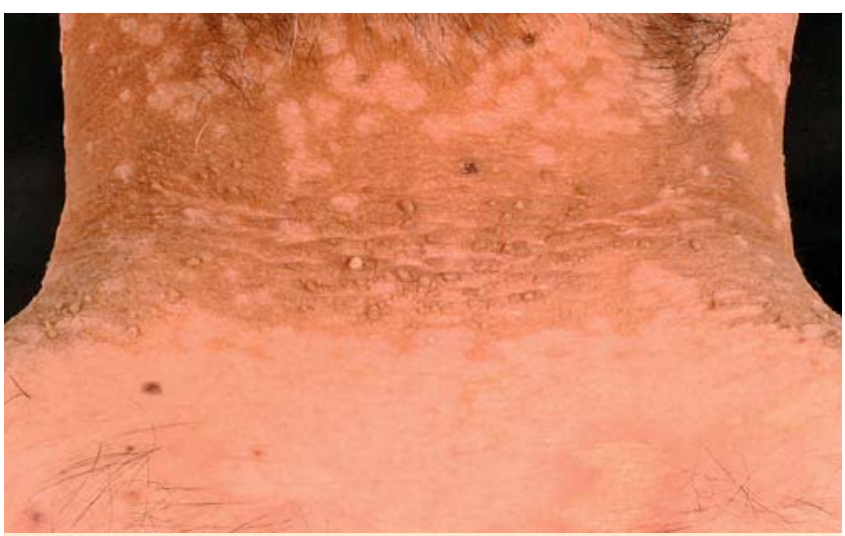

Abb.3 Akanthosis nigricans mit Hyperpigmentierung, Lichenifikation und kleinen Papeln.

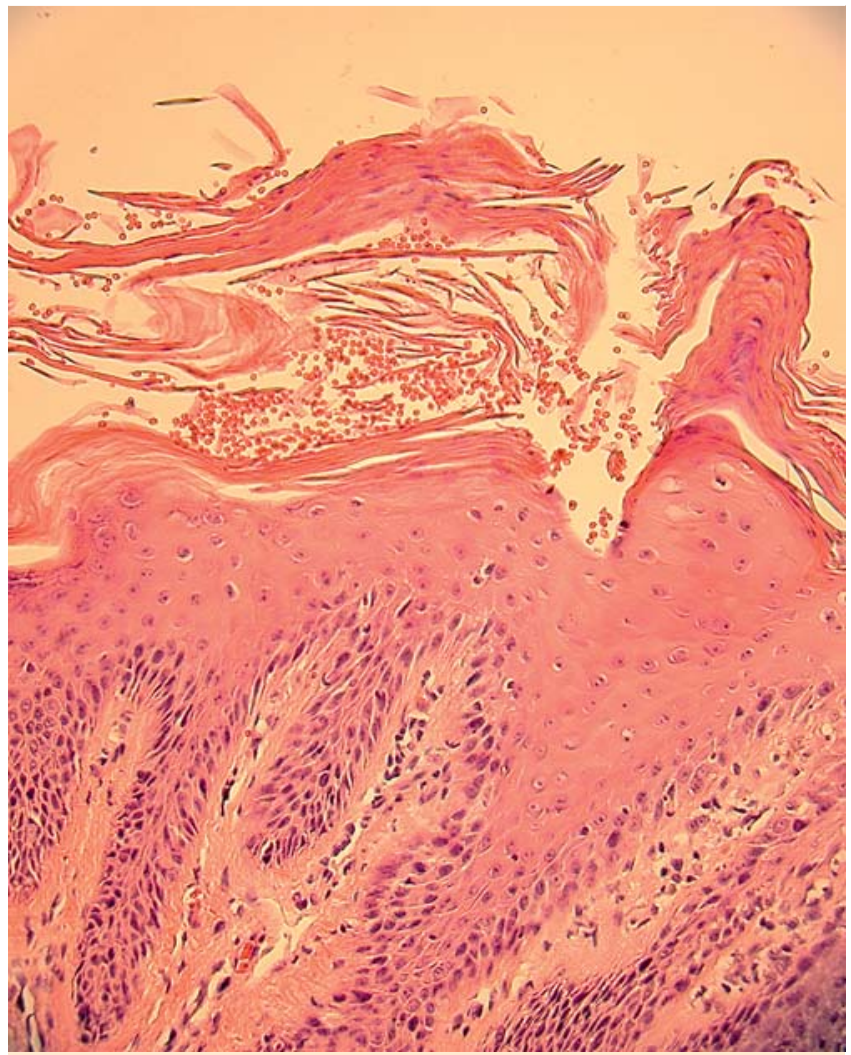

Abb.4 Pityriasis rubra pilaris: psoriasiforme Akanthose, follikuläre Hyperkeratose und "Schulterparakeratosen“.

läre Hyperkeratose, eine ausgeprägte Schulterparakeratose (ohne Munro-Mikroabszesse), im Bereich des Epithels eine psoriasiforme Akanthose sowie subepithelial entzündliche Veränderungen mit lympho-histiozytärem Infiltrat.

Probe 2 hingegen $(\bullet$ Abb.5), Entnahmestelle axillär, war durch eine mächtige Orthohyperkeratose mit angedeuteten Pseudohornzysten sowie durch eine gleichmäßige Akanthose mit basaler Hyperpigmentierung gekennzeichnet, subepithelial kaum Entzündung.

Unter der klinischen Verdachtsdiagnose einer Pityriasis rubra pilaris, welche im Verlauf des stationären Aufenthalts histopathologisch bestätigt wurde, starteten wir bereits am Aufnahmetag mit der Gabe von Methotrexat 7,5 mg mit tags darauf folgender Folsäuresubstitution von $5 \mathrm{mg}$. Danach regelmäßige Gabe von Methotrexat $15 \mathrm{mg}$ immer am Samstag sowie $5 \mathrm{mg}$ Folsäure immer sonntags. Als Lichttherapie führten wir 4-mal in der Woche eine Bade-PUVA-Therapie durch. Als Lokaltherapie erhielt der Patient für Rumpf und Extremitäten morgens Betamethason 0,1\% in Kühlsalbe, mittags Chinosol $2 \%$ in Kühlsalbe, abends harnstoffhaltige Kerasal-Basis-Salbe. Im Bereich von Gesicht, Ohren und Hals morgens und mittags Schwefel-Zink-Paste sowie abends Ketoconazol-Creme. Für den Kopfbereich schließlich morgens Volon-A-Tinktur (Triamcinolon) und Celestan-V-Creme (Betamethason), mittags Ketoconazol-Creme, zusätzlich Lichttherapie mit UV-Kamm.

Darunter kam es zu einer langsam schleppenden, jedoch nachhaltigen Besserung, die Herde blassten ab. Von einer rein symptomatischen Behandlung der ebenfalls histopathologisch nachgewiesenen Acanthosis nigricans sahen wir bei fehlendem subjektiven Leidensdruck des Patienten ab. 


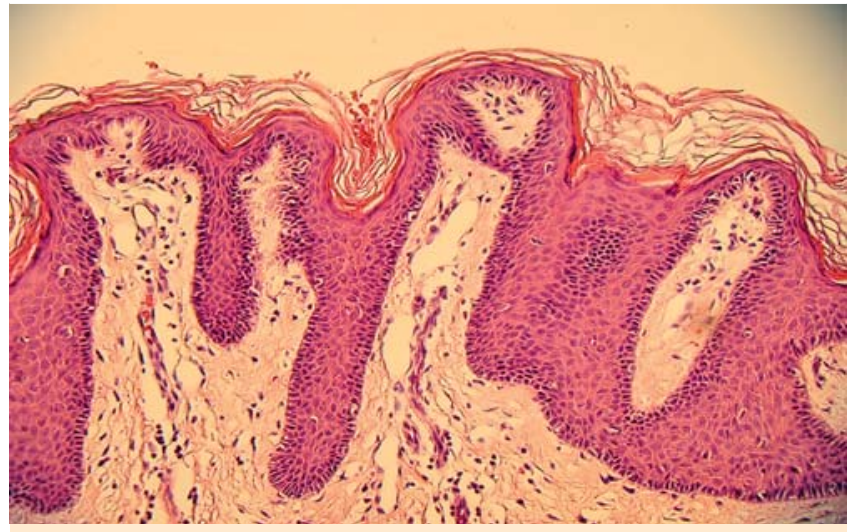

Abb.5 (Pseudo-)Akanthosis nigricans. Orthohyperkeratose mit kleinen Pseudohornzysten, schmalzapfige Akanthose, basale Hyperpigmentierung, wenig Entzündung.

\section{Diskussion}

$\nabla$

Die Pityriasis rubra pilaris ist eine seltene, meist chronisch verlaufende Erkrankung unklarer Ätiologie [6,9], welche durch eine Palmoplantarhyperkeratose und follikuläre Keratosen gekennzeichnet ist sowie nicht selten mit einer Erythrodermie einhergeht $[1,13]$. Erstmanifestationen treten in jedem Lebensalter [3], insbesondere jedoch in der ersten und fünften Lebensdekade auf $[1,6]$. Betroffen sind, insbesondere in der Initialphase, die Extremitätenstreckseiten sowie im weiteren Verlauf vor allem das Capillitium, das Gesicht und der Rumpf, jedoch auch Handinnenflächen und Fußsohlen. Sie ist gekennzeichnet durch ein typisches Reibeisengefühl, einen orange-roten bis lachsfarbenen Farbton sowie charakteristische Aussparungen befallener Haut, auch bei großflächigem Befall, den sogenannten „nappes claires“ [1]. Histopathologisch zeigen sich eine kräftige Hyperkeratose mit der charakteristischen perifollikulären Schulterparakeratose, im Bereich des Epithels eine psoriasiforme Akanthose sowie in der Dermis ein lympho-histiozytäres Infiltrat. Die wichtigsten Differenzialdiagnosen sind die Psoriasis, Ekzeme sowie kutane Lymphome, wobei insbesondere die Unterscheidung zur Psoriasis oft diffizil ist [2]. Die Therapie dieser Erkrankung gestaltet sich grundsätzlich schwierig, mühsam und langwierig und stellt somit nicht selten eine große Herausforderung dar $[1,3,12,13]$. Topisch kommen kurzfristig Kortikosteroide, langfristig keratolytische und rehydratisierende Externa zum Einsatz. Als Lichttherapie wird die PUVA-Therapie angewandt. Als systemische Optionen stehen systemische Retinoide [1,12], Ciclosporin sowie insbesondere bei schweren Verlaufsformen Methotrexat zur Verfügung [6]. In Einzelfällen wurde auch schon über die sehr erfolgreiche Gabe von Fumaraten und TNF-alpha-blockierenden Biologika berichtet $[2,3,10,12]$, die uns aber nicht in jedem Fall notwendig erscheinen. Systemische Kortikosteroide erwiesen sich hingegen als ineffektiv [3]. Kürzlich konnten für die familiäre Variante der PRP Mutationen im Gen für CARD 14 nachgewiesen werden. Dieses Protein ist für die NF-kappa-B vermittelte Regulation des epidermalen Epithels wichtig und Polymorphismen dieses Gens konnten auch bei Psoriasispatienten gefunden werden [14].

Bei der Acanthosis nigricans handelt es sich um eine Hyperpigmentierung und Papillomatose in den großen Körperfalten. Man unterscheidet dabei grundsätzlich zwischen einer paraneoplastischen Form („Acanthosis nigricans maligna“) und verschiedenen nicht-paraneoplastischen Formen („Pseudoacanthosis nigri- cans“). Die paraneoplastische Form, welche etwa 25\% aller Fälle ausmacht, zeichnet sich durch ein rasches Auftreten mit starker Ausprägung und häufig auch dem Befall der Mundschleimhaut aus $[4,8]$. Die mit Abstand häufigsten assoziierten Malignome sind intraabdominelle Tumoren, davon wiederum am häufigsten Adenokarzinome des Magens [8]. Die nicht-paraneoplastischen Formen beruhen meist auf endokrinologischen Störungen, vor allem auf Insulin-resistenten Formen des Diabetes mellitus [4, 11] und, besonders häufig, Bestehen einer Adipositas $[4,7,11]$. Sie können aber auch hereditär bedingt oder Medikamenten-induziert [4] sein. Schließlich treten auch idiopathische Varianten auf. Die Aktivierung von epidermalen Keratinozyten und dermalen Fibroblasten durch Wachstumsfaktoren wird als wichtige Komponente in der Pathogenese der Erkrankung angesehen [4]. Histopathologisch zeigen sich eine Orthohyperkeratose, teilweise Pseudohornzysten, die an eine seborrhoische Keratose erinnern, sowie eine Akanthose, basale Hyperpigmentierung sowie nur geringes entzündliches Infiltrat in der Dermis, wobei die paraneoplastische und nicht-paraneoplastische Form keine histologischen Unterschiede aufweisen. Besonders wichtig bei diesem dermatologischen Erscheinungsbild ist eine genaue Ursachenabklärung, insbesondere müssen Malignome konsequent ausgeschlossen werden. Die Therapie besteht primär aus der Beseitigung einer möglichen Ursache. Daneben stehen rein symptomatische Maßnahmen wie konsequente Hygiene der Intertrigines, austrocknende und keratolytische Externa sowie in schweren Fällen ggf. systemische Retinoide sowie operative Optionen [5] zur Verfügung.

Bei unserem Patienten wurde initial die Diagnose „Psoriasis“ gestellt, was bei der Pityriasis rubra pilaris nicht selten vorkommt [12]; entsprechend wurde er jahrelang auf eine Psoriasis behandelt. Unter dem schweren Krankheitsbild, das der Patient bei Aufnahme präsentierte, starteten wir neben einer Bade-PUVA-Therapie auch eine systemische Methotrexattherapie, welche ihre volle Wirksamkeit oft erst nach Monaten entfaltet. Dementsprechend empfahlen wir die konsequente langfristige Fortführung beider Therapien im ambulanten Bereich. Die Acanthosis nigricans war dem Patienten bis zum Aufnahmezeitpunkt nicht auffällig. Diesbezüglich war uns neben der histopathologischen Diagnosesicherung vor allem eine gründliche Tumorsuche zum Ausschluss einer paraneoplastischen Form wichtig. Dabei traten weder laborchemisch noch bildmorphologisch Malignom-verdächtige Befunde zutage, eine bereits ambulant durchgeführte Gastroskopie war ebenfalls unauffällig. Wir empfahlen dem Patienten dringend, auch ambulant regelmäßige Tumorkontrollen durchführen zu lassen. Eine wahrscheinliche Ursache der Erkrankung liegt in dem deutlichen Übergewicht des Patienten, das ja auch mit einem leicht erhöhten HbA1c von 6,1\% verknüpft war. Entsprechend sollte der Patient ambulante Maßnahmen zur Gewichtsreduktion ergreifen.

Eine Beschreibung dieser Kombination der beiden Erkrankungen konnten wir trotz intensiver Recherche nicht finden, sodass unser Patient eine Erstbeschreibung darstellt. Wir sehen aber keinen inhaltlichen Zusammenhang zwischen beiden Erkrankungen.

\section{Interessenkonflikt}

$\nabla$

Die Autoren geben an, dass keine Interessenkonflikte bestehen. 


\section{Abstract}

\section{Pityriasis rubra pilaris in Combination with Acanthosis nigricans \\ $\nabla$}

We report on a 53-years old male patient, who was treated since more than 12 years with the diagnosis "psoriasis" and who presented himself with a suberythrodermia with boarder marked plaques with discrete scales especially at trunk and extremities with areas of healthy skin. Additionally, a lichenification of the skin-relief with fibrinous papules and black hyperpigmentation in the neck, axillary and inguinal including perianal could be noted. We clinically diagnosed a pityriasis rubra pilaris as well as an (pseudo)acanthosis nigricans. Both diagnoses could be substantiated by histology. We successfully started a therapy with methotrexat, ultraviolet irradiation with bath-PUVA and an intensive local therapy. To the best of our knowledge, this combination of the two diseases was not mentioned in the literature until now.

\section{Literatur}

1 Artik S, Megahed M, Ruzicka T. Pityriasis rubra pilaris: Fallberichte und Übersicht der Literatur. Der Hautarzt 2003; 54: 858 - 863

2 Coras B, Vogt T, Ulrich $H$ et al. Fumaric acid esters therapy: a new treatment modality in pityriasis rubra pilaris? British Journal of Dermatology $2005 ; 152: 388-389$

3 Gemmeke A, Schönlebe J, Koch A et al. Pityriasis rubra pilaris - a retrospective single center analysis over eight years. Journal der Deutschen Dermatologischen Gesellschaft 2010; 8: 439-444

4 Hartman R, DeFelice T, Tzu J et al. Acanthosis nigricans in the setting of niacin therapy. Dermatology Online Journal 2011; 17: 11

5 Kapoor S. Diagnosis and treatment of Acanthosis nigricans. Skinmed 2010; 8: $161-164$

6 Klein A, Landthaler M, Karrer S. Pityriasis rubra pilaris: a review of diagnosis and treatment. Am J Clin Dermatol 2010; 11: 157-170

7 Kong AS, Williams RL, Rhyne R et al. Acanthosis nigricans: high prevalence and association with diabetes in a practice-based research network consortium - a PRImary care Multi-Ethic Network study. J Am Board Fam Med 2010; 23: 476-485

8 Lenzner $U$, Ramsauer J, Petzoldt $W$ et al. Acanthosis nigricans maligna: Fallbeschreibung und Literaturübersicht. Der Hautarzt 1998; 49: 41 47

9 Magro CM, Crowson AN. The clinical and histomorphological features of pityriasis rubra pilaris. Journal of Cutaneous Pathology 1997; 24: 416-424

10 Müller H, Gattringer C, Zelger B et al. Infliximab monotherapy as firstline treatment for adult-onset pityriasis rubra pilaris: Case report and review of the literature on biologic therapy. Journal of the American Academy of Dermatology 2008; 59: 65-70

11 Pinheiro AC, Rojas P, Carrasco F et al. Acanthosis nigricans as an indicator of insulin resistence in Chilean adult population. Nutricion Hospitaliaria 2011; 26: 940-944

12 Seckin D, Tula E, Ergun T. Successful use of etanercept in type I pityriasis rubra pilaris. British Journal of Dermatology 2007; 158: 642 - 644

13 Sehgal VN, Srivastava G, Dogra S. Adult onset pityriasis rubra pilaris. Indian J Dermatol Venereol Leprol 2008; 74: 311 - 321

14 Fuchs-Telem D, Sarig $O$, van Steensel MA et al. Familial pityriasis rubra pilaris is caused by mutations in CARD14. Am J Hum Genet 2012; 91: 163-170 